\title{
Two-Grid Iterative Method for a Class of Fredholm Functional Integral Equations based on the Radial Basis Function Interpolation
}

\author{
Qisheng Wang ${ }^{1 *}$ and Huimin Zhou ${ }^{1}$ \\ ${ }^{1}$ School of Mathematics and Computational Science, Wuyi University, Jiangmen, Guangdong, P.R. China \\ *Corresponding author E-mail: 282228006@qq.com
}

\author{
Article Info \\ Keywords: Fredholm functional inte- \\ gral equations, The radial basis func- \\ tion interpolation, Two-grid iterative \\ method \\ 2010 AMS: 65D30, 45D05, 45G10 \\ Received: 16 October 2019 \\ Accepted: 08 December 2019 \\ Available online: 20 December 2019
}

\begin{abstract}
In this paper, we discuss a two-grid iterative method for solving a class of Fredholm functional integral equations based on the radial basis function interpolation. Firstly, the existence and uniqueness of the solution are proved by Banach fixed point theorem. Secondly, the algorithm and convergence analysis of the radial basis function approximation method is given on the coarse grid. Thirdly, the fine grid iterative solution and convergence results are obtained. Finally, the validity and reliability of the theoretical analysis are verified by two numerical experiments.
\end{abstract}

\section{Introduction}

Integral equations are widely used in quantum physics, engineering design, astronomy, geography, biomedicine and other fields, so it is of great application value to explore the solution of integral equations.

For a long time, the algorithms of integral equations have been widely concerned and studied. Many different methods have been used to approximate the solutions of some integral equations [1, 2]. In recent years, F.Muller and W.Varnhorn [3] have studied approximation and numerical solution of Fredholm integral equations of second kind using quasi-interpolation. Some convergence analysis for 2-dimensional Fredholm integral equation with complex factors by Meshless method were introduced in [4]. Application of Legendre wavelets for solving a class of functional integral equations were discussed in [5]. Chelyshkov collocation approach was developed in [6] for solving linear weakly singular Volterra integral equations. In addition, we know that the computational complexity of numerical integral discretization depends on the diameter $h$ of mesh generation. The calculated workload is usually $O\left(n^{3}\right)$, where $n=1 / h$. Therefore, the construction of a suitable two-grid algorithm can solve the difficulty of computational complexity. Two-grid method is a discretization technique based on two meshes of different sizes, which has been concerned by many researchers [7]-[10] for a long time. Therefore, it is very necessary to enrich the efficient algorithms of different types of integral equations.

In this paper, we consider the following as a class of Fredholm functional integral equations

$$
u(x)-A(x) u(\alpha(x))=f(x)+\lambda \int_{\Omega} k(x, s) u(s) d s, x \in \Omega,
$$

where $\Omega$ is the bounded closed area in $R^{d}, d=1,2,3, x=\left(x_{1}, x_{2}, \cdots, x_{d}\right), s=\left(s_{1}, s_{2}, \cdots, s_{d}\right), \alpha(x)=\left(\alpha_{1}(x), \alpha_{2}(x), \cdots, \alpha_{d}(x)\right)$, and $A(x), \alpha(x), f(x), k(x, s)$ are properly smooth known functions, $u(x) \in R$ is the unknown function.

The contents of this article are as follows. Section 2 contains the proofs of the existence and uniqueness of the exact solution for (1.1). In section 3, we discuss the radial basis function approximation method and convergence results on the coarse grid for (1.1). Section 4, is devoted to the two-grid iterative method and convergence results on the fine grid for (1.1). In the last section, the correctness of the theory is proved by two numerical examples. 


\section{The existence and uniqueness of solution}

In this section, the conditions which provides the existence and uniqueness of exact solution of (1.1) are given by using Banach fixed point theorem.

Theorem 2.1. Assume that $\Omega$ is a suitable Banach space and $A(x), \alpha(x), f(x) \in C(\Omega), k(x, s) \in C(\Omega \times \Omega)$. If the following conditions are satisfied

$$
\left\{\begin{array}{l}
(i) \alpha(x) \in \Omega, \text { for } x \in \Omega \\
(i i)\|A(x)\|_{\infty}+|\lambda| \cdot\left\|\int_{\Omega}|k(x, s)| d s\right\|_{\infty}=\gamma<1
\end{array}\right.
$$

where $\|\cdot\|_{\infty}=\max |\cdot|$. Then the (1.1) has a unique solution.

Proof. Let $T$ be a mapping from $C(\Omega)$ to $C(\Omega)$ with

$$
T u(x)=A(x) u(\alpha(x))+f(x)+\lambda \int_{\Omega} k(x, s) u(s) d s,
$$

for $u \in C(\Omega)$. Let $u_{1}, u_{2}$ be two solutions of (1.1), then we have

$$
\begin{aligned}
\left\|T u_{1}-T u_{2}\right\|_{\infty} & =\left\|A(x)\left[u_{1}(\alpha(x))-u_{2}(\alpha(x))\right]+\lambda \int_{\Omega} k(x, s)\left[u_{1}(s)-u_{2}(s)\right] d s\right\|_{\infty} \\
& \leq\left(\|A(x)\|_{\infty}+|\lambda| \cdot\left\|\int_{\Omega}|k(x, s)| d s\right\|_{\infty}\right) \cdot\left\|u_{1}-u_{2}\right\|_{\infty} \\
& \leq \gamma \cdot\left\|u_{1}-u_{2}\right\|_{\infty} .
\end{aligned}
$$

Note that $0<\gamma<1$, by the Banach fixed point theorem, then $T$ is a contractive mapping on $\left(C(\Omega)\right.$, $\left.\|\cdot\|_{\infty}\right)$. So there exists the unique solution $u^{*} \in C(\Omega)$ such that $T u^{*}=u^{*}$, and (1.1) has a unique solution.

\section{The radial basis function interpolation and convergence on the coarse grid}

In this section, we give the algorithm of the radial basis function interpolation for (1.1), and obtain the convergence theorem in the infinite norm sense.

First of all, Assume $\left\{x^{i}\right\}_{i=1}^{m}, x^{i}=\left(x_{1}^{i}, x_{2}^{i}, \cdots, x_{d}^{i}\right) \in \Omega$ is a series of irregular observation points of $u(x)$ on $\Omega$, and let the basis of the radial basis function is $\varphi_{1}(x), \varphi_{2}(x), \cdots, \varphi_{m}(x)$, where $\varphi_{i}(x)=\varphi\left(\left\|x-x^{i}\right\|_{2}\right), i=1,2, \cdots, m$. Note that $r=\left\|x-x^{i}\right\|_{2}$. There are three common radial basis functions (see [11]):

(1)Gaussian distribution function $\varphi(r)=e^{-a^{2} r^{2}}$;

(2)MQ function $\varphi(r)=\left(c^{2}+r^{2}\right)^{b}$ and IMQ function $\varphi(r)=\left(c^{2}+r^{2}\right)^{-b}(b>0)$;

(3)Thin plate splines function $\varphi(r)=r^{2 k-d} \log r$.

Now, we construct vector space $V_{m}(\Omega)=\operatorname{span}\left\{\varphi_{1}(x), \varphi_{2}(x), \cdots, \varphi_{m}(x)\right\}$. And let the radial basis function interpolation $u_{m}^{I}(x)=\sum_{i=1}^{m} c_{i} \varphi_{i}(x) \in$ $V_{m}$, satisfying $u_{m}^{I}\left(x^{i}\right)=u\left(x^{i}\right), i=1,2, \cdots, m$. From [11], we can get the following error estimation between the radial basis function and the exact solution:

$$
\left\|u(x)-u_{m}^{I}(x)\right\|_{\infty} \leq \operatorname{ch}^{\frac{e}{2}}
$$

where $h=\sup _{x \in \Omega} \min _{1 \leq i \leq m}\left\|x-x^{i}\right\|$.

Therefore, let $u(x)=u_{m}^{I}(x)+\varepsilon_{m}(x)$ where $\varepsilon_{m}(x)$ is the interpolation remainder of $u(x)$ on $V_{m}(\Omega)$. Then we can obtain

$$
u_{m}^{I}(x)+\varepsilon_{m}(x)-A(x)\left[u_{m}^{I}(\alpha(x))+\varepsilon_{m}(\alpha(x))\right]=f(x)+\lambda \int_{\Omega} k(x, s)\left[u_{m}^{I}(s)+\varepsilon_{m}(s)\right] d s .
$$

Ignore the error terms $\varepsilon_{m}(x)$ and $\varepsilon_{m}(\alpha(x))$ and substitute $u_{m}^{I}(x)=\sum_{i=1}^{m} c_{i} \varphi_{i}(x)$ into (3.1), we get the approximate equation of (1.1):

$$
\sum_{i=1}^{m} c_{i} \varphi_{i}(x)-A(x) \sum_{i=1}^{m} c_{i} \varphi_{i}(\alpha(x))=f(x)+\sum_{i=1}^{m} c_{i} \lambda \int_{\Omega} k(x, s) \varphi_{i}(s) d s .
$$

Remove the items then we have

$$
\sum_{i=1}^{m} c_{i}\left[\varphi_{i}(x)-A(x) \varphi_{i}(\alpha(x))-\lambda \int_{\Omega} k(x, s) \varphi_{i}(s) d s\right]=f(x)
$$

Let $\psi_{i}(x)=\varphi_{i}(x)-A(x) \varphi_{i}(\alpha(x))-\lambda \int_{\Omega} k(x, s) \varphi_{i}(s) d s, \quad i=1,2, \cdots, m$, then (3.2) can be written as

$$
\sum_{i=1}^{m} c_{i} \psi_{i}(x)=f(x)
$$

Since $\varphi_{1}(x), \varphi_{2}(x), \cdots, \varphi_{m}(x)$ are linear independent functions on $V_{m}(\Omega)$ and satisfies the condition of Theorem 2.1, which implies $\psi_{1}(x), \psi_{2}(x), \cdots, \psi_{m}(x)$ are also linear independent functions on $V_{m}(\Omega)$. Here's the proof.

Because

$$
\sum_{i=1}^{m} c_{i} \psi_{i}(x)=\sum_{i=1}^{m} c_{i} \varphi_{i}(x)-A(x) \sum_{i=1}^{m} c_{i} \varphi_{i}(\alpha(x))-\lambda \int_{\Omega} k(x, s) \sum_{i=1}^{m} c_{i} \varphi_{i}(s) d s
$$


and

$$
\left\|\sum_{i=1}^{m} c_{i} \varphi_{i}(\alpha(x))\right\|_{\infty} \leq\left\|\sum_{i=1}^{m} c_{i} \varphi_{i}(x)\right\|_{\infty}, \quad \alpha(x) \in \Omega .
$$

From Theorem 2.1 and trigonometric inequality we can get

$$
(1-\gamma) \cdot\left\|\sum_{i=1}^{m} c_{i} \varphi_{i}(x)\right\|_{\infty} \leq\left\|\sum_{i=1}^{m} c_{i} \psi_{i}(x)\right\|_{\infty} \leq(1+\gamma) \cdot\left\|\sum_{i=1}^{m} c_{i} \varphi_{i}(x)\right\|_{\infty}
$$

For $x \in \Omega$, assume that $\sum_{i=1}^{m} c_{i} \psi_{i}(x)=0$, then we obtain $\left\|\sum_{i=1}^{m} c_{i} \psi_{i}(x)\right\|_{\infty}=0$. From (3.4) we have $\left\|\sum_{i=1}^{m} c_{i} \varphi_{i}(x)\right\|_{\infty}=0$. Therefore,

$$
\sum_{i=1}^{m} c_{i} \varphi_{i}(x)=0
$$

And because $\varphi_{1}(x), \varphi_{2}(x), \cdots, \varphi_{m}(x)$ are linear independent functions on $V_{m}(\Omega)$, so

$$
c_{1}=c_{2}=\cdots=c_{n}=0 .
$$

The proof is complete.

(3.3) take the collocation points $x=x^{j}=\left(x_{1}^{j}, x_{2}^{j}, \cdots, x_{d}^{j}\right), j=1,2, \cdots, m$, then we get $\sum_{i=1}^{m} c_{i} \psi_{i}\left(x^{j}\right)=f\left(x^{j}\right)$. Written in matrix form is

$$
G_{m} \cdot C_{m}=F_{m},
$$

where $C_{m}=\left(c_{1}, c_{2}, \cdots, c_{m}\right)^{T}, F_{m}=\left(f\left(x^{1}\right), f\left(x^{2}\right), \cdots, f\left(x^{m}\right)\right)^{T}, G_{m}=\left(g_{i j}\right)_{m \times m}, g_{i j}=\psi_{i}\left(x^{j}\right), i, j=1,2, \cdots, m$.

Let $G_{m}$ is a nonsingular matrix, then $G_{m}^{-1}$ exists. So there is

$$
C_{m}^{*}=G_{m}^{-1} \cdot F_{m}=\left(c_{1}^{*}, c_{2}^{*}, \cdots, c_{m}^{*}\right)^{T} .
$$

Therefore, $u_{m}^{*}(x)=\sum_{i=1}^{m} c_{i}^{*} \varphi_{i}(x)$ is called the approximate solution of radial basis function of (1.1).

Theorem 3.1. Assume that $u(x)$ is the exact solution and $u_{m}^{*}(x)$ is the approximate solution of radial basis function, then

$$
\left\|u-u_{m}^{*}\right\|_{\infty} \leq \operatorname{ch}^{\frac{e}{2}}+\sum_{i=1}^{m}\left\|\varphi_{i}(x)\right\|_{\infty} \cdot\left\|G_{m}^{-1}\right\|_{\infty} \cdot\left\|R_{m}(\varepsilon)\right\|_{\infty},
$$

where $R_{m}(\varepsilon)=-\varepsilon_{m}(x)+A(x) \varepsilon_{m}(\alpha(x))+\lambda \int_{\Omega} k(x, s) \varepsilon(s) d s$, and for $\forall x \in \Omega$, we have

$$
\lim _{h \rightarrow 0} u_{m}^{*}(x)=u(x) \text {. }
$$

Proof. For (3.1) and $u_{m}^{I}(x)=\sum_{i=1}^{m} c_{i} \varphi_{i}(x)$, we can get

$$
\sum_{i=1}^{m} c_{i} \varphi_{i}(x)+\varepsilon_{m}(x)-A(x)\left[\sum_{i=1}^{m} c_{i} \varphi_{i}(\alpha(x))+\varepsilon_{m}(\alpha(x))\right]=f(x)+\lambda \int_{\Omega} k(x, s)\left[\sum_{i=1}^{m} c_{i} \varphi_{i}(s)+\varepsilon_{m}(s)\right] d s,
$$

abbreviated in the following form

$$
\sum_{i=1}^{m} c_{i} \psi_{i}(x)=f(x)+\left[-\varepsilon_{m}(x)+A(x) \varepsilon_{m}(\alpha(x))+\lambda \int_{\Omega} k(x, s) \varepsilon_{m}(s) d s\right] .
$$

Now we write down the above equation as

$$
\sum_{i=1}^{m} c_{i} \psi_{i}(x)=f(x)+R_{m}(\varepsilon)
$$

where $R_{m}(\varepsilon)=-\varepsilon_{m}(x)+A(x) \varepsilon_{m}(\alpha(x))+\lambda \int_{\Omega} k(x, s) \varepsilon_{m}(s) d s$. The corresponding matrix form is

$$
G_{m} \cdot C_{m}=F_{m}+R_{m}(\varepsilon) .
$$

Subtracting (3.5) from (3.6)to get $G_{m}\left(C_{m}-C_{m}^{*}\right)=R_{m}(\varepsilon)$, so

$$
\left\|C_{m}-C_{m}^{*}\right\|_{\infty} \leq\left\|G_{m}^{-1}\right\|_{\infty} \cdot\left\|R_{m}(\varepsilon)\right\|_{\infty} .
$$

Then for

$$
\begin{aligned}
\left\|u-u_{m}^{*}\right\|_{\infty} & =\left\|u-u_{m}^{I}+u_{m}^{I}-u_{m}^{*}\right\|_{\infty} \\
& \leq\left\|u-u_{m}^{I}\right\|_{\infty}+\left\|u_{m}^{I}-u_{m}^{*}\right\|_{\infty} \\
& \leq c h^{\frac{e}{2}}+\left\|\sum_{i=1}^{m}\left(c_{i}-c_{i}^{*}\right) \varphi_{i}(x)\right\|_{\infty} \\
& \leq c h^{\frac{e}{2}}+\sum_{i=1}^{m} \mid c_{i}-c_{i}^{*}\left\|\varphi_{i}(x)\right\|_{\infty} \\
& \leq c^{\frac{e}{2}}+\sum_{i=1}^{m}\left\|\varphi_{i}(x)\right\|_{\infty} \cdot\left\|G_{m}^{-1}\right\|_{\infty} \cdot\left\|R_{m}(\varepsilon)\right\|_{\infty} .
\end{aligned}
$$

Since $\left\|R_{m}(\varepsilon)\right\|_{\infty} \rightarrow 0,\left\|u-u_{m}^{*}\right\|_{\infty} \rightarrow 0$. 


\section{Two-grid iterative method and convergence analysis}

As we all know, for the discretization of numerical integration of (1.1), the computational complexity depends on the diameter $h$ of mesh subdivision, and the workload of numerical calculation is usually $O\left(n^{3}\right)$, where $n=1 / h$. Therefore, computational complexity can be well solved by constructing an appropriate two-layer grid algorithm.

In this section, a new fixed point iterative approximation method is introduced in three steps to obtain two-grid iterative solution on the fine grid. The iterative algorithm is as follows

Algorithm 1.

Step 1. Select a series of irregular observation points of $u(x)$ on the fine grid as $\left(y^{1}, y^{2}, \cdots, y^{n}\right), y^{i}=\left(y_{1}^{i}, y_{2}^{i}, \cdots, y_{d}^{i}\right), i=1,2, \cdots, n, d=$ 1,2 or 3 .

Step 2. Approximate solution of Radial basis function on the fine grid is

$$
u_{n}^{(0)}(x)=\sum_{i=1}^{n} l_{i} \Phi_{i}(x)
$$

where $\Phi_{i}(x)=\varphi\left(\left\|x-y^{i}\right\|\right), i=1,2, \cdots, n$.

Step 3. Take the initial value $u_{n}^{(0)}(x)=u_{m}^{*}(x)$ and construct iterative scheme

$$
u_{n}^{(k+1)}(x)=A(x) u_{n}^{(k)}(\alpha(x))+f(x)+\lambda \int_{\Omega} k(x, s) u_{n}^{(k)}(s) d s, k=0,1,2, \cdots
$$

The error estimation and convergence results between the fine grid approximate solution of radial basis function $u_{n}^{I}(x)$ and the two-grid iterative solution $u_{n}^{(k+1)}(x)$ are given below.

Theorem 4.1. Let $u_{n}^{I}(x)$ is the fine grid approximate solution of radial basis function and $u_{n}^{(k+1)}(x)$ is the $(k+1)$ th iterative solution determined by (4.1), then

$$
\left\|u_{n}^{I}(x)-u_{n}^{(k+1)}(x)\right\|_{\infty} \leq \gamma^{k+1} \cdot\left\|u_{n}(x)-u_{n}^{(0)}(x)\right\|_{\infty}
$$

where $\gamma<1$ as in Theorem 2.1.

For $\forall x \in \Omega$, there holds

$$
\lim _{k \rightarrow \infty} u_{n}^{(k)}(x)=u_{n}^{I}(x)
$$

Proof. Replace $u_{n}^{I}(x)=\sum_{i=1}^{n} c_{i} \varphi_{i}(x)$ in (1.1) and get

$$
u_{n}^{I}(x)=A(x) u_{n}^{I}(\alpha(x))+f(x)+\lambda \int_{\Omega} k(x, s) u_{n}^{I}(s) d s
$$

Subtracting (4.1) from (4.2), we have

$$
u_{n}^{I}(x)-u_{n}^{(k+1)}(x)=A(x)\left[u_{n}^{I}(\alpha(x))-u_{n}^{(k)}(\alpha(x))\right]+\lambda \int_{\Omega} k(x, s)\left[u_{n}^{I}(s)-u_{n}^{(k)}(s)\right] d s,
$$

then

$$
\begin{aligned}
\left\|u_{n}^{I}(x)-u_{n}^{(k+1)}(x)\right\|_{\infty} & =\left\|A(x)\left[u_{n}^{I}(\alpha(x))-u_{n}^{(k)}(\alpha(x))\right]+\lambda \int_{\Omega} k(x, s)\left[u_{n}^{I}(s)-u_{n}^{(k)}(s)\right] d s\right\|_{\infty} \\
& \leq\left(\|A(x)\|_{\infty}+|\lambda| \cdot\left\|\int_{\Omega}|k(x, s)| d s\right\|_{\infty}\right) \cdot\left\|u_{n}^{I}-u_{n}^{(k)}\right\|_{\infty} \\
& \leq \gamma \cdot\left\|u_{n}^{I}-u_{n}^{(k)}\right\|_{\infty} .
\end{aligned}
$$

In this way,

$$
\left\|u_{n}^{I}(x)-u_{n}^{(k+1)}(x)\right\|_{\infty} \leq \gamma^{k+1} \cdot\left\|u_{n}^{I}-u_{n}^{(0)}\right\|_{\infty}
$$

can be obtained by progressive recursion.

From Theorem 2.1, we get $0<\gamma<1$, such that

$$
\lim _{k \rightarrow \infty} \gamma^{k+1}=0
$$

For $x \in \Omega$, there exists

$$
\lim _{k \rightarrow \infty} u_{n}^{(k)}(x)=u_{n}^{I}(x)
$$




\section{Numerical examples}

In this section, two numerical examples $(d=2)$ are given to illustrate the feasibility and validity of the above algorithm and its convergence analysis. The exact solution is compared with the two-grid iterative solution and its error estimation in the infinite norm sense is provided by using Matlab 2015a.

Selecting $\{(m, n)\}=\{(8,32),(8,64),(16,64)\}$, we calculate the maximum error $\left\|u_{n}(x, y)-u_{n}^{(k)}(x, y)\right\|_{\infty}=\max _{\left(x_{i}, y_{i}\right) \in \Omega}\left|u_{n}\left(x_{i}, y_{i}\right)-u_{n}^{(k)}\left(x_{i}, y_{i}\right)\right|$ between the fine grid radial basis interpolation solution $u_{n}(x, y)$ and two-grid iterative solution $u_{n}^{(k)}(x, y)$ with $\{k\}=\{3,4,5,6\}$.

Example 5.1. Consider the following Fredholm functional integral equation

$$
u(x, y)-\frac{x+y}{16} u\left(\alpha_{1}(x), \alpha_{2}(y)\right)=f(x, y)+\frac{1}{20} \int_{\Omega}(x+y) u\left(s_{1}, s_{2}\right) d s_{1} d s_{2},
$$

where $\Omega=\left\{(x, y) \mid 0 \leq x \leq 1,0 \leq y \leq x^{2}\right\}, \alpha_{1}(x)=\frac{1}{4} x, \alpha_{2}(x)=\frac{1}{16} x^{2}$ and $f(x, y)=x y-\frac{1}{240} x-\frac{1}{240} y-\frac{1}{16} x y^{2}\left(\right.$ fracx $\left.64+\frac{y}{64}\right)$. (5.1) has an exact solution $u(x, y)=x y$.

We solve (5.1) by two-grid method based on radial basis interpolation, and our experimental results can be seen from Table 1 . The results were obtained by using Gaussian distribution function $(a=1)$ and IMQ function $(c=\sqrt{2}, b=1)$, respectively. Next, the exact solution $u(x, y)$ and the two-grid iterative solution $u_{n}^{(k)}(x, y)$ which are given and can be seen from Figure 5.1(a) and Figure 5.1(b) while $(m, n, k)=(8,64,6)$.

\begin{tabular}{|c|c|c|c|c|c|c|c|c|c|c|c|c|c|c|}
\hline \multirow[b]{2}{*}{$\mathrm{m}$} & \multirow[b]{2}{*}{$\mathrm{n}$} & \multirow[b]{2}{*}{$\mathrm{k}$} & \multicolumn{2}{|c|}{$\left\|u_{n}-u_{n}^{(k)}\right\|_{\infty}$} & \multirow[b]{2}{*}{$\mathrm{m}$} & \multirow[b]{2}{*}{$\mathrm{n}$} & \multirow[b]{2}{*}{$\mathrm{k}$} & \multicolumn{2}{|c|}{$\left\|u_{n}-u_{n}^{(k)}\right\|_{\infty}$} & \multirow[b]{2}{*}{$\mathrm{m}$} & \multirow[b]{2}{*}{$\mathrm{n}$} & \multirow[b]{2}{*}{$\mathrm{k}$} & \multicolumn{2}{|c|}{$\left\|u_{n}-u_{n}^{(k)}\right\|_{\infty}$} \\
\hline & & & Gaussian & IMQ & & & & Gaussian & IMQ & & & & Gaussian & IMQ \\
\hline 8 & 32 & 3 & $9.1698 \mathrm{e}-04$ & $1.3462 \mathrm{e}-04$ & 8 & 64 & 3 & $1.5243 \mathrm{e}-03$ & $9.3253 e-04$ & 16 & 64 & 3 & $1.2412 \mathrm{e}-05$ & $7.5523 \mathrm{e}-06$ \\
\hline & & 4 & $2.2979 \mathrm{e}-05$ & $2.1310 \mathrm{e}-05$ & & & 4 & $2.4130 \mathrm{e}-04$ & $1.4762 \mathrm{e}-04$ & & & 4 & $1.9648 \mathrm{e}-06$ & $1.1955 \mathrm{e}-06$ \\
\hline & & 5 & $1.9648 \mathrm{e}-06$ & $3.3734 \mathrm{e}-06$ & & & 5 & $3.8198 \mathrm{e}-05$ & $2.3368 \mathrm{e}-05$ & & & 5 & $3.1103 \mathrm{e}-07$ & $1.8925 \mathrm{e}-07$ \\
\hline & & 6 & $5.7583 \mathrm{e}-07$ & $5.3401 \mathrm{e}-07$ & & & 6 & $6.0467 \mathrm{e}-06$ & $3.6992 \mathrm{e}-06$ & & & 6 & $4.9236 \mathrm{e}-08$ & $2.9958 \mathrm{e}-08$ \\
\hline
\end{tabular}

Table 1: The maximum error $\left\|u_{n}(x)-u_{n}^{(k)}(x)\right\|_{\infty}$ for Example 5.1.

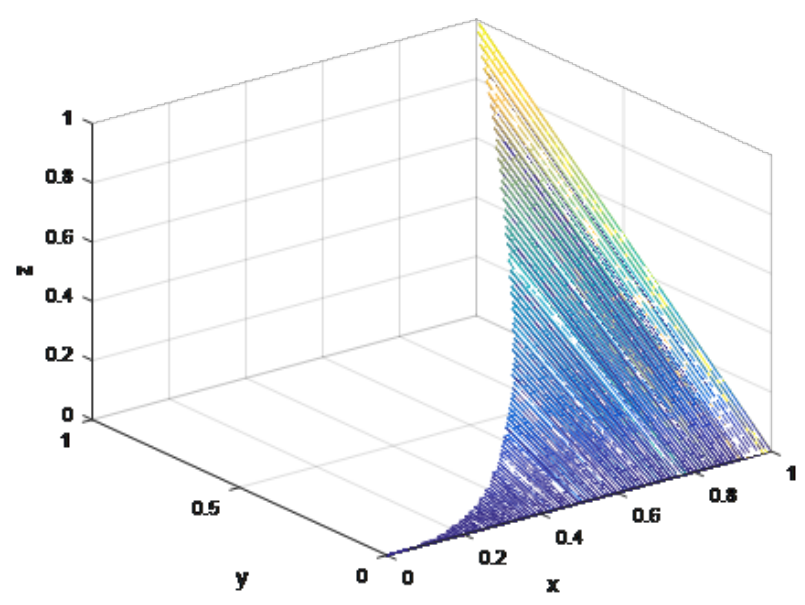

(a) The exact solution $u(x, y)$ of example 5.1

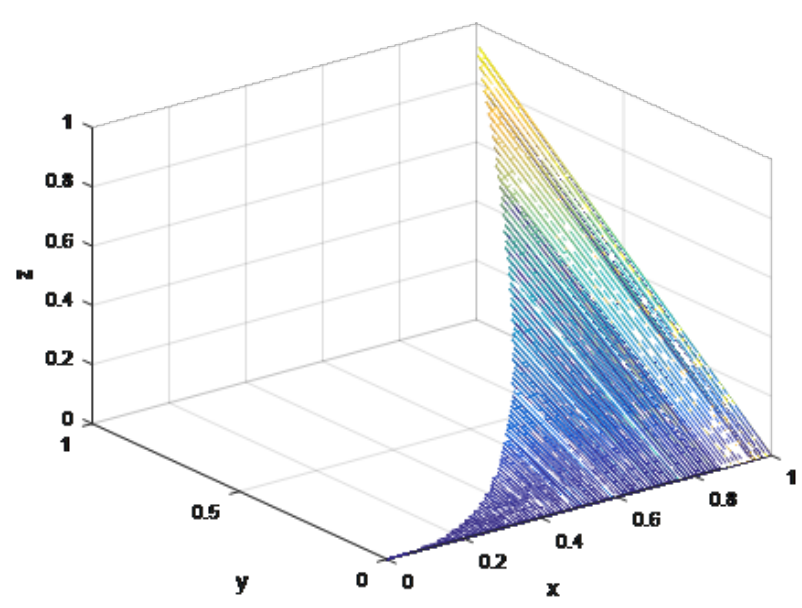

(b) The two-grid iterative solution $u_{n}^{(k)}(x, y)$ of example 5.1

Example 5.2. Consider the following Fredholm functional integral equation

$$
u(x, y)-\frac{1}{10} e^{x y} u\left(\alpha_{1}(x), \alpha_{2}(y)\right)=f(x, y)+\frac{1}{10} \int_{\Omega} x y s_{1} s_{2} u\left(s_{1}, s_{2}\right) d s_{1} d s_{2}
$$

where $\Omega=\{(x, y) \mid 0 \leq x \leq 1,0 \leq y \leq x\}, \alpha_{1}(x)=x, \alpha_{2}(x)=\frac{1}{2} x$ and $f(x, y)=e^{x+y}-\frac{1}{10} e^{x+\frac{y}{2}} e^{x y}-\frac{x y}{20}$. (5.2) has an exact solution $u(x, y)=e^{x+y}$.

We also solve (5.2) by two-grid method based on radial basis interpolation, and the experimental results can be seen from Table 2 . The results were obtained by using Gaussian distribution function $(a=1)$ and MQ function $\left(c=4, b=\frac{1}{2}\right)$, respectively. Next, the exact solution $u(x, y)$ and the two-grid iterative solution $u_{n}^{(k)}(x, y)$ which are given and can be seen from Figure 5.1(c) and Figure 5.1(d) while $(m, n, k)=(8,64,6)$. 


\begin{tabular}{|c|c|c|c|c|c|c|c|c|c|c|c|c|c|c|}
\hline \multirow[b]{2}{*}{$\mathrm{m}$} & \multirow[b]{2}{*}{$\mathrm{n}$} & \multirow[b]{2}{*}{$\mathrm{k}$} & \multicolumn{2}{|c|}{$\left\|u_{n}-u_{n}^{(k)}\right\|_{\infty}$} & \multirow[b]{2}{*}{$\mathrm{m}$} & \multirow[b]{2}{*}{$\mathrm{n}$} & \multirow[b]{2}{*}{$\mathrm{k}$} & \multicolumn{2}{|c|}{$\left\|u_{n}-u_{n}^{(k)}\right\|_{\infty}$} & \multirow[b]{2}{*}{$\mathrm{m}$} & \multirow[b]{2}{*}{$\mathrm{n}$} & \multirow[b]{2}{*}{$\mathrm{k}$} & \multicolumn{2}{|c|}{$\left\|u_{n}-u_{n}^{(k)}\right\|_{\infty}$} \\
\hline & & & Gaussian & IMQ & & & & Gaussian & IMQ & & & & Gaussian & IMQ \\
\hline 8 & 32 & 3 & $5.3422 \mathrm{e}-03$ & $1.5322 \mathrm{e}-03$ & 8 & 64 & 3 & $8.7424 \mathrm{e}-03$ & $1.6395 \mathrm{e}-03$ & 16 & 64 & 3 & $4.4892 \mathrm{e}-04$ & $9.7401 \mathrm{e}-05$ \\
\hline & & 4 & $1.6000 \mathrm{e}-03$ & $4.5476 \mathrm{e}-04$ & & & 4 & $2.6000 \mathrm{e}-03$ & $4.8660 \mathrm{e}-04$ & & & 4 & $1.3324 \mathrm{e}-04$ & $2.8909 \mathrm{e}-05$ \\
\hline & & 5 & $4.7488 \mathrm{e}-04$ & $1.3497 \mathrm{e}-04$ & & & 5 & $7.7168 \mathrm{e}-04$ & $1.4442 \mathrm{e}-04$ & & & 5 & $3.9546 \mathrm{e}-05$ & $8.5802 \mathrm{e}-06$ \\
\hline & & 6 & $1.4094 \mathrm{e}-04$ & $4.0059 \mathrm{e}-05$ & & & 6 & $2.2903 \mathrm{e}-04$ & $4.2864 \mathrm{e}-05$ & & & 6 & $1.1737 \mathrm{e}-05$ & $2.5466 \mathrm{e}-06$ \\
\hline
\end{tabular}

Table 2: The maximum error $\left\|u_{n}(x)-u_{n}^{(k)}(x)\right\|_{\infty}$ for Example 5.2.

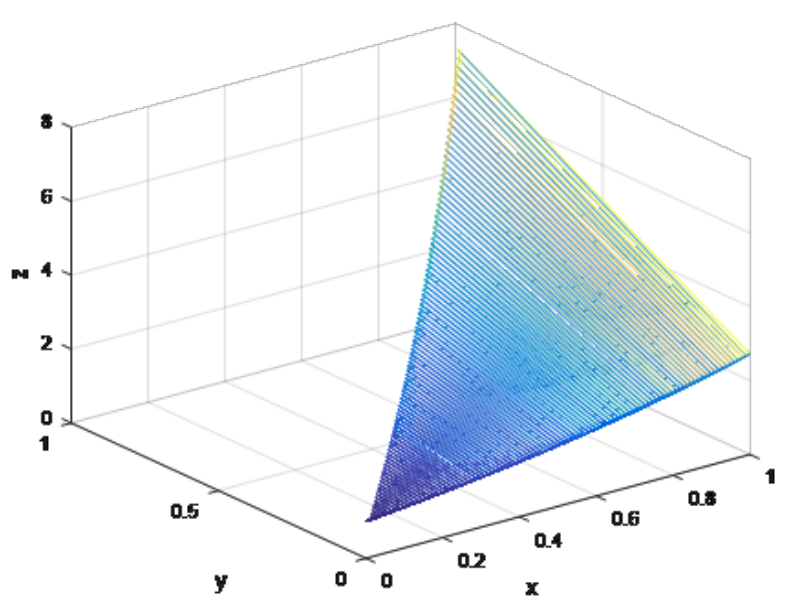

(c) The exact solution $u(x, y)$ of example 5.2

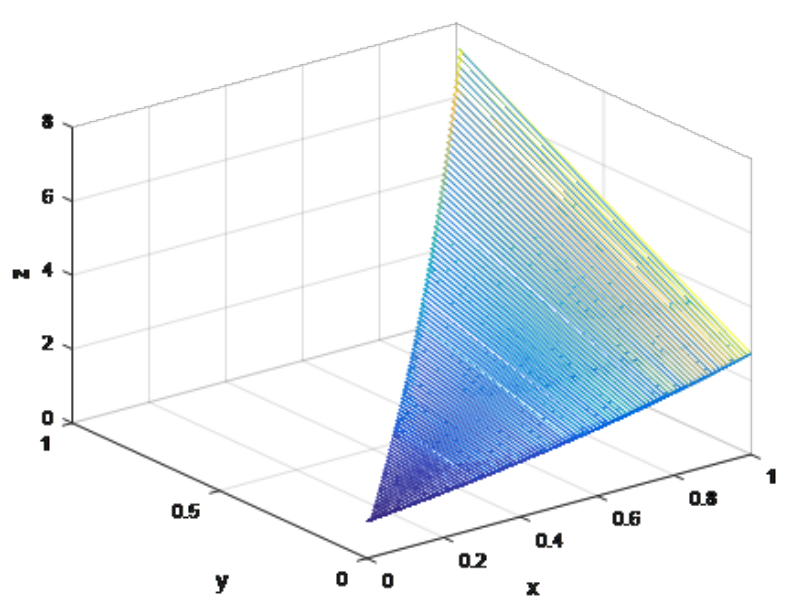

(d) The two-grid iterative solution $u_{n}^{(k)}(x, y)$ of example 5.2

\section{Conclusion}

In this paper, a new two-grid method based on the radial basis function interpolation for solving a class of Fredholm functional integral equations, which has practical value is presented. The algorithm and convergence analysis of two-grid iterative solution are given. At the same time, the method can greatly reduce the computational workload. Our numerical results can successfully prove the correctness of the proposed error estimation. Extending this method to other integral equations is our further research.

\section{Acknowledgement}

This research was supported by the Natural Science Fundation of Guangdong Province(Grant No.2015A030313643,2016A030313005), Postgraduate Demonstration Course Construction Project of Guangdong Province(Grant No.2018SFKC38) and Postgraduate Demonstration Course Construction Project of Wuyi University(Grant No.YJS-SFKC-18-01).

\section{References}

[1] K. E. Atkinson, W. Han, Theoretical Numerical Analysis, 2nd edn. Springer, Berlin, (2005).

[2] K. E. Atkinson, Iterative methods for the numerical solution of Fredholm integral equations of the second kind, Technical Report, Computer Center, Australian Natl. Univ., Canberra.

[3] F. Muller, W. Varnhorn, On approximation and numerical solution of Fredholm integral equations of second kind using quasi-interpolation, Appl. Math. Comput., 217 (2011), 6409-6416.

[4] Q. S. Wang, H. S. Wang, Meshless method and convergence analysis for 2-dimensional Fredholm integral equation with complex factors, J. Comput. Appl. Math., 304 (2016), 18-25.

[5] M. Felahat, M. M. Moghadam, A. A. Askarihemmat, Application of Legendre wavelets for solving a class of functional integral equations, Iran. J. Sci. Technol., 43(3) (2019), 1089-1100.

[6] Y. Talaei, Chelyshkov collocation approach for solving linear weakly singular Volterra integral equations, J. Appl. Math. Comput., 60(1-2) (2019), 201-222.

[7] K. E. Atkinson, Two-grid iteration methods for linear integral equations of the second kind on piecewise smooth surfaces in R3, SIAM J. Sci Comput., 15(5) (1994), 1083-1104.

[8] C. Chen, W. Liu, A two-grid method for finite element solutions for nonlinear parabolic equations, Abstr. Appl. Anal., 2012(11) (2012).

[9] J. Yan, Q. Zhang, L. Zhu, Z. Zhang, Two-grid methods for finite volume element approximations of nonlinear Sobolev equations, Numer. Funct. Anal. Optim., 37 (2016), 391-414.

[10] C. J. Chen, K. Li, Y. P. Chen, Y. Q. Huang, Two-grid finite element methods combined with Crank-Nicolson scheme for nonlinear Sobolev equations, Adv. Comput. Math., 45 (2019), 611-630.

[11] Z. M. Wu, Convergence analysis for the Radial basis function interpolation, Ann. Math., 14A(4) (1993), 480-486. (in Chinese). 